\title{
Stability Properties of Network Diversity Multiple Access with Multiple-Antenna Reception and Imperfect Collision Multiplicity Estimation
}

\author{
Ramiro Samano-Robles and Atilio Gameiro \\ Instituto de Telecomunicações, Campus Universitário, 3810-193 Aveiro, Portugal \\ Correspondence should be addressed to Ramiro Samano-Robles; ramiro@av.it.pt
}

Received 23 March 2012; Accepted 15 December 2012

Academic Editor: Yang Yang

Copyright (c) 2013 R. Samano-Robles and A. Gameiro. This is an open access article distributed under the Creative Commons Attribution License, which permits unrestricted use, distribution, and reproduction in any medium, provided the original work is properly cited.

\begin{abstract}
In NDMA (network diversity multiple access), protocol-controlled retransmissions are used to create a virtual MIMO (multipleinput multiple-output) system, where collisions can be resolved via source separation. By using this retransmission diversity approach for collision resolution, NDMA is the family of random access protocols with the highest potential throughput. However, several issues remain open today in the modeling and design of this type of protocol, particularly in terms of dynamic stable performance and backlog delay. This paper attempts to partially fill this gap by proposing a Markov model for the study of the dynamic-stable performance of a symmetrical and non-blind NDMA protocol assisted by a multiple-antenna receiver. The model is useful in the study of stability aspects in terms of the backlog-user distribution and average backlog delay. It also allows for the investigation of the different states of the system and the transition probabilities between them. Unlike previous works, the proposed approach considers the imperfect estimation of the collision multiplicity, which is a crucial process to the performance of NDMA. The results suggest that NDMA improves not only the throughput performance over previous solutions, but also the average number of backlogged users, the average backlog delay and, in general, the stability of random access protocols. It is also shown that when multiuser detection conditions degrade, ALOHA-type backlog retransmission becomes relevant to the stable operation of NDMA.
\end{abstract}

\section{Introduction}

1.1. NDMA and Cross-Layer Design in Random Access. The last two decades have witnessed the proliferation of advanced random-access protocols assisted by signal processing tools [1]. In these novel algorithms, spatial, code, or frequency resources are conveniently exploited in order to enable the simultaneous reception of more than one packet at the physical layer (PHY). Random-access protocols based on this innovative PHY layer have been termed multipacket reception (MPR) protocols [2]. In the design of MPR protocols, the conventional collision model (where collisions imply the loss of all the contending packets) is no longer useful [2]. A new approach that considers the co-design of medium access control (MAC) and PHY layers (MAC-PHY crosslayer design) is thus required $[3,4]$.
A breakthrough in the literature of MAC-PHY crosslayer design was the work in [5], which proposed a new approach to achieve diversity in random access by exploiting retransmissions in the time domain. The new protocol, coined network diversity multiple access (NDMA), creates a receive diversity source by dynamically requesting from the contending users as many retransmissions as necessary to resolve collisions via source separation. Therefore, in NDMA, packets with collision are not discarded as in the conventional protocols. Instead, they are stored in memory for further processing. Initially, the system uses the stored signals with collision to calculate the collision multiplicity (i.e., the number of contending users). The system then proceeds to request further retransmissions (which are also to be stored in memory) from the contending users, until the collected signals mimic a MIMO (multiple-input multiple-output) 
system. The collision can then be potentially resolved via source separation if the number of diversity sources is at least equal to the number of contending signals. In NDMA, MAC-PHY cross-layer design plays a more crucial role than that in conventional MPR protocols: PHY-layer diversity is explicitly created by retransmissions controlled by the MAC layer. In addition, the estimation of the required number of retransmissions depends on PHY layer processing to determine the collision-multiplicity. The performance of NDMA critically depends on this estimation process. Training-based versions have been proposed for non-dispersive and dispersive channels in $[5,6]$, respectively. Two blind versions, one for slow-fading channels with source separation based on rotational invariance techniques and another one for fastfading channels with source separation based on independent component analysis, have been proposed in $[7,8]$, respectively. Other modeling approaches for NDMA have been proposed in $[9,10]$.

1.2. Open Issues and Paper Contributions. Despite recent advances in the study of NDMA, several issues remain open today, particularly in terms of stability analysis. Stability in random access can be briefly defined here as the ability to control/empty the queues of all users within a finite period of time. Stability of NDMA in asymmetrical environments has been studied in the ideal case of perfect estimation of the collision multiplicity and perfect packet reception in [11]. An outer bound for the stability region of NDMA in asymmetrical environments has been derived in [12] for the case of imperfect estimation of the collision multiplicity. This derivation assumed that packets incorrectly received are dropped from the system; that is, backlog traffic is not considered. This assumption, however, is valid only in scenarios with high signal-to-noise ratio (SNR). Therefore, a lot of work is still required to analyze the backlog traffic of NDMA in scenarios with finite SNR with imperfect collision multiplicity estimation and imperfect packet reception.

To address these issues, this paper proposes a novel Markov model for stability, throughput, and backlog traffic analysis of symmetrical and non-blind NDMA protocols assisted by MPR. This work assumes imperfect estimation of the collision multiplicity with a packet reception model given by the performance of the user detector. All the analysis work presented in this paper is carried out by assuming scenarios with finite SNR. The analysis consists of two initial steps: (1) definition of the backlog states that describe the network at any given time, which in this case are given by the numbers of backlogged users, and (2) calculation of the transition probabilities between network states using channel and queuing statistics. The steady-state probabilities of the different network states are then mapped into a one-dimensional state vector. Similarly, each transition probability constitutes an element of a transition probability matrix. This state vector and transition probability matrix define a Markov model that can be solved by conventional eigenvalue analysis. Each user is thus modeled in two possible states: idle or backlog. A user being in the backlog state means that it has previously transmitted a packet that was incorrectly detected by the receiver. The system performance is then evaluated for different values of packet transmission probability when users are in the idle state, and different values of the packet retransmission probability when the users are in the backlog state. The results in this paper show that NDMA with MPR provides a considerable improvement on stability over conventional collision-resolution algorithms. In addition, it is shown that ALOHA-type backlog retransmission schemes become relevant to the stability of NDMA when the multiuser detection conditions decline due to low SNR and/or due to a low number of receiver antennas (i.e., weak MPR).

1.3. Paper Structure. The remainder of this paper is organized as follows. Section 2 describes the system model. Section 3 provides the performance model for user detection and collision multiplicity estimation. Section 4 describes the proposed metrics and the Markov model. Section 5 presents the discussion of the results obtained by plotting the expressions derived in previous sections. Finally, Section 6 draws the conclusions of the paper.

\section{System Model}

2.1. Scenario Description and NDMA Epoch-Slot Definition. Consider the slotted random access network depicted in Figure 1 with a set of $J$ buffered users and one central node or base station (BS) with $M$ receiver antennas that will be used for purposes of multipacket reception and user detection. The channel between user $j$ and the $m$ th antenna of the BS is denoted by $h_{j, m}$. All channels are assumed to be non-dispersive, block-fading, and independently and identically distributed (i.i.d.) with Rayleigh statistics: $h_{j, m} \sim \mathscr{C} \mathscr{N}\left(0, \sigma_{a}^{2}\right)$.

Whenever a terminal with a packet in its buffer is allowed to transmit, it proceeds to do so at the beginning of a time-slot. Since NDMA exploits the time domain to create diversity, the number of time slots used to resolve any packet collision will be a random variable that will be denoted here by $l$. The period of time used to resolve a packet collision will be called contention resolution period or epoch-slot (see Figure 1). For convenience in the analysis, two types of epoch slots are defined: a relevant epoch, which is the epoch where a given user under analysis participates by transmitting a packet, and an irrelevant epoch, which is the epoch where a given user under analysis does not participate (no packet transmission). Their lengths will be denoted here by $l_{r}$ and $l_{i r}$, respectively. For example, in Figure 1, the first epoch is relevant to user 1 but irrelevant to user 5 . By contrast, the second epoch in Figure 1 is relevant to all the users displayed in the figure.

2.2. Signal Model for User Detection. Each user of the network is preassigned with a unique orthogonal code with $J$ symbols $\mathbf{w}_{j}=\left[w_{j}(0), \ldots, w_{j}(J-1)\right]^{T}$, where $(\cdot)^{T}$ is the vector transpose operator. This code, which is attached as a header of each packet transmission, will be used for purposes of 


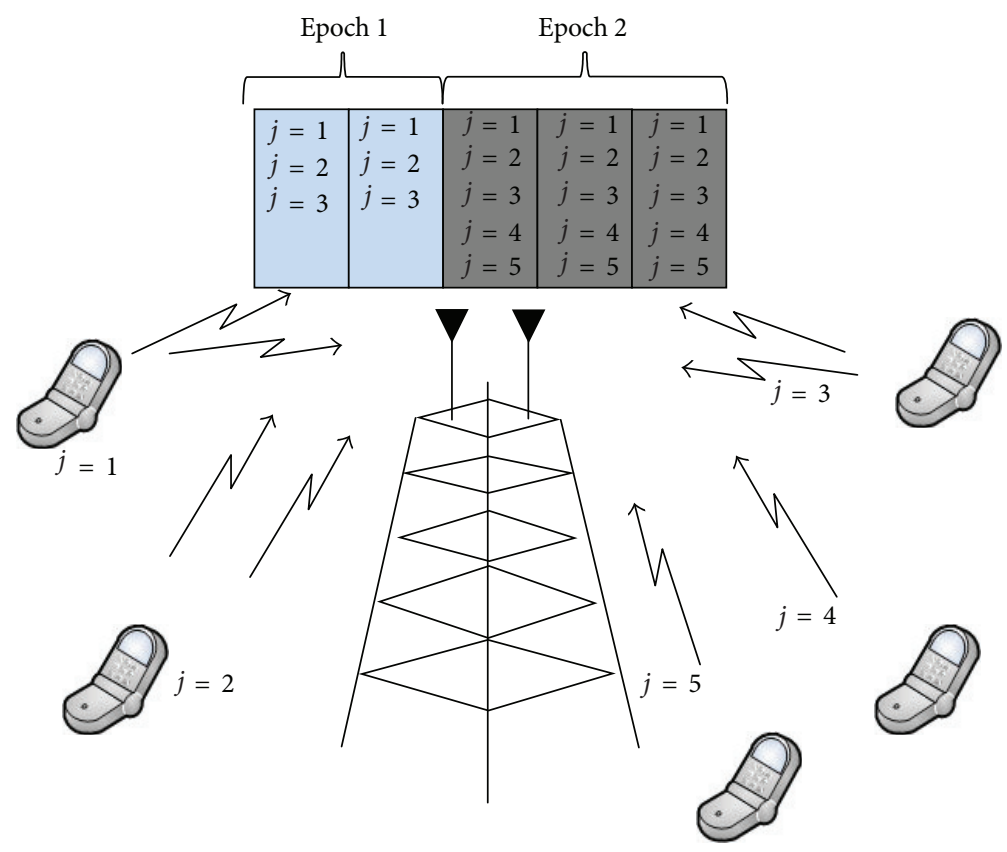

FIGURE 1: Random-access network assisted by retransmission diversity and multipacket reception.

user detection and channel estimation [5]. The orthogonality condition of the set of codes is given by

$$
\mathbf{w}_{j}^{T} \mathbf{w}_{k}= \begin{cases}J, & k=j \\ 0, & k \neq j .\end{cases}
$$

The received signal header at the $m$ th antenna, which is formed by the superposition of all the headers of all the transmissions of the set of contending users (denoted here by $\mathscr{T})$, can be written as follows:

$$
\mathbf{y}_{m}^{(h)}=\sum_{j \in \mathscr{T}} h_{j, m} \mathbf{w}_{j}+\mathbf{v}_{m}^{(h)}
$$

where $\mathbf{v}_{m}^{(h)}=\left[v_{m}^{(h)}(0), \ldots, v_{m}^{(h)}(J-1)\right]^{T}$ is the zero-mean and circularly white complex gaussian noise vector in the received header. This means that $v_{m}^{(h)}(r) \sim \mathscr{C} \mathscr{N}\left(0, \sigma_{v}^{2}\right)$, where $r \in\{0, \ldots, J-1\}$. The BS uses a matched-filter operation $\left(\mathbf{w}_{j}^{T} \mathbf{y}_{m}^{(h)}\right)$ to extract the detection statistics of user $j$ from each antenna. The results from all the antennas are then combined as follows:

$$
z_{j}=\sum_{m=1}^{M}\left|\mathbf{w}_{j}^{T} \mathbf{y}_{m}^{(h)}\right|^{2}
$$

The total detection statistic $z_{j}$ for user $j$ is then compared to a detection threshold $\beta$ to decide whether user $j$ is active or not. If $z_{j}<\beta$, then the user is detected as inactive: $j \notin \widehat{\mathscr{T}}$, where $\widehat{\mathscr{T}}$ is the estimated set of contending users. Otherwise, if $z_{j}>\beta$, then the user is detected as active $(j \in \widehat{\mathscr{T}})$. Since this detection process is prone to errors due to fading and noise, two cases of potential active user detection can be identified: (1) user $j$ can be correctly detected as active with probability $P_{D}$ (probability of correct detection) provided the user has transmitted a packet and (2) user $j$ is incorrectly detected as active with probability $P_{F}$ (probability of false alarm) provided the user did not transmit a packet. By detecting the presence each one of the contending users, the BS can also have an estimation $\widehat{K}=|\widehat{\mathscr{T}}|$ of the real collision multiplicity $K=|\mathscr{T}|$, where $|\cdot|$ is the set cardinality operator when applied to a set variable.

2.3. Protocol Operation and Signal Model for MPR. At the beginning of every epoch slot, the BS proceeds to estimate the collision multiplicity as described in the previous subsection. The estimation of the collision multiplicity can be improved by exploiting the new retransmissions received in subsequent time slots within the collision resolution period. This type of detection is known as sequential user detection [5]. This type of detector is, however, out of our scope of this paper, and thus we will focus on the conventional user detection considering only the first received transmission at the beginning of the collision resolution period as in [5]. Once the BS has obtained an estimation $\widehat{K}$ of the collision multiplicity $K$, then it proceeds to calculate the number of retransmissions required to resolve the collision. Since the BS has $M$ receiver antennas, the number of transmissions (including the initial transmission plus retransmissions) required in the non-blind version of NDMA is given by $\lceil\widehat{K} / M\rceil$, where $\lceil\cdot\rceil$ is the ceil integer operator. This means that the number of diversity sources (time and space) must be greater than or equal to the estimated collision multiplicity. In Figure 1, we can observe two realizations of epochs with $M=2$ antennas at the BS. In the first epoch, three users have collided in the first time slot. Since only two antennas are provided 
and three signals need to be recovered, then only one more retransmission is needed to potentially resolve the collision. In this case, the combined number of collected transmissions and space resources (antennas) is equal to four, which is enough to attempt the recovery of the three contending signals. The second epoch experiences five contending users, which requires two retransmissions (six diversity sources) to be resolved. Having more diversity sources than contending signals is necessary to ensure that the channel matrix is full rank, which in turn improves the probability of success of the source separation technique to be used [5]. To request a retransmission for diversity purposes, the BS simply indicates with a feedback flag at the end of each time slot to all the contending users that retransmission is required in the next time slot. The feedback flag is kept on until all necessary retransmissions have been collected. This feedback channel is assumed to be ideal. All the collected (re)transmissions are kept in memory to create a MIMO system that can be expressed as follows $[5,11]$ :

$$
\mathbf{Y}_{(M\lceil\widehat{K} / M\rceil) \times N}=\mathbf{A}_{(M\lceil\widehat{K} / M\rceil) \times K} \mathbf{S}_{K \times N}+\mathbf{W}_{(M\lceil\widehat{K} / M\rceil) \times N},
$$

where $\mathbf{Y}$ is the array formed by the collection of all received signals from all the $M$ antennas and from the $\lceil\widehat{K} / M\rceil$ time slots of the epoch, $\mathbf{A}$ is the mixing matrix or MIMO channel, $\mathbf{S}$ is the array of stacked packets from all the contending users, each one with $N$ symbols, and finally $\mathbf{W}$ is the collected Gaussian noise components. The mixing matrix A can be estimated by using the outcome of the matched filter operation from each antenna. The estimate $\widehat{\mathbf{A}}$ can be used to recover the contending packets using a zero forcing equalizer, $\widehat{\mathbf{S}}=\left(\widehat{\mathbf{A}}^{H} \widehat{\mathbf{A}}\right)^{-1} \widehat{\mathbf{A}}^{H} \mathbf{Y}$, or a minimum mean square error (MMSE) receiver, $\widehat{\mathbf{S}}=\left(\widehat{\mathbf{A}}^{H} \widehat{\mathbf{A}}+\sigma_{v}^{2} \mathbf{I}\right)^{-1} \widehat{\mathbf{A}}^{H} \mathbf{Y}$, where the term $\widehat{\mathbf{S}}$ indicates the soft estimate of the contending packets. This signal is then passed through a hard symbol detection stage to try to obtain the original packets $\mathbf{S}$ [5].

To facilitate NDMA MAC layer design, packet reception performance is usually approximated by the outcome of the collision multiplicity estimation [5]. If all the contending users are correctly detected as active and none of the noncontending uses are incorrectly detected as active (false alarm), then all the packets are considered as correctly received by the BS. Otherwise, in the case of any detection error, all packets are assumed to be lost in the collision resolution process. The BS also indicates to all the contending users, by means of an ideal feedback flag at the end of a resolution period, whether the resolution process was successful or not. All packets that were incorrectly detected enter a back-off retransmission process that is described in following sections. These protocol steps are repeated for subsequent epoch slots.

\section{Detector Performance Model}

NDMA depends critically upon the performance of the user detector [12]. This section deals with the modeling of the user detector in (3). This will be useful for subsequent calculations and design of the MAC layer. The probability of false alarm of a user that did not transmit a signal while still being detected as active can be defined more formally as follows:

$$
P_{F}=\operatorname{Pr}\left\{z_{j}>\beta \mid j \notin \mathscr{T}\right\},
$$

which is the probability that the detection statistic $z_{j}$ is above the detection threshold $\beta$, conditional on user $j$ not being one of the contending users. Since noise is Gaussian distributed, the detection statistic $z_{j}$ in (3), which consists of the summation of $2 M$ squared i.i.d. zero-mean gaussian signals, can be easily proved to have for this particular case a central chi-square distribution with $2 M$ degrees of freedom and parameter $J \sigma_{v}^{2}$. The term $J \sigma_{v}^{2}$ comes from the matched-filter operation in (3), which combines $J$ different noise signals (coming from each one of the $J$ symbols of the header). Therefore, the probability of false alarm $P_{F}$ can be expressed as the complementary cumulative distribution function (CCDF) of the central chi-squared distribution with $2 M$ degrees of freedom [13]:

$$
P_{F}=e^{-\left(\beta / J \sigma_{v}^{2}\right)} \sum_{m=0}^{M} \frac{1}{m !}\left(\frac{\beta}{J \sigma_{v}^{2}}\right)^{m} \text {. }
$$

Similarly, the probability of correct detection of user $j$, conditional on user $j$ being one of the contending users, can be defined as

$$
P_{D}=\operatorname{Pr}\left\{z_{j}>\beta \mid j \in \mathscr{T}\right\} .
$$

Since both channel and noise are Gaussian distributed; the detection statistic $z_{j}$ in (3), which is given by the summation of $2 M$ squared i.i.d. zero-mean gaussian signals, can also be proved for this particular case to have a central chi-square distribution with $2 M$ degrees of freedom and parameter $J^{2} \sigma_{a}^{2}+J \sigma_{v}^{2}$. The term $J^{2} \sigma_{a}^{2}$ comes from the matched-filter operation in (3), which considers the channel term $J$ times. The second term $J \sigma_{v}^{2}$ has the same explanation as in the case of the probability of false alarm in (5). Therefore, the probability of correct detection $P_{D}$ is also given by the CCDF of the central chi-squared distribution with $2 M$ degrees of freedom. This means that $P_{D}$ can be obtained by replacing in (6) the term $J \sigma_{v}^{2}$ with $J^{2} \sigma_{a}^{2}+J \sigma_{v}^{2}$. This concludes the definition of the receiver operational curve (ROC) of the user detector.

\section{Markov Model and Performance Metrics}

4.1. Markov Model and Backlog State Distribution. To investigate the stability and dynamic properties of the system, each user will be assumed to be in two possible states: idle or backlog (see Figure 2). The backlog state means that the user has transmitted a packet in a previous epoch slot and its reception has failed. Therefore, the user is waiting to retransmit the packet with probability $p_{r}$. When a user is in the backlog state, no new incoming packets in the queue are allowed to be transmitted [14]. By contrast, when the user is in the idle state, a new incoming packet in the buffer is allowed to be transmitted with probability $p_{a}$. The total traffic arrival process (new incoming and backlog traffic) of 
each user will be assumed to have a Poisson distribution with parameter $\lambda$. The state of the network at any epoch slot is given by the number of users in backlog state, denoted here by $n$ (see Figure 3 ).

The transition probability between two states in two consecutive epoch slots will be denoted by $P_{n, i}$, which is the probability of having $i$ backlogged users in the next epoch slot given that in the current epoch $n$ users are in the backlog state. This is illustrated in Figure 3. By assuming that all packets are correctly received when all the contending users are correctly detected as active and none of the non-contending users incurs in a false alarm, the transition probabilities can be calculated by identifying three different cases: (1) when the number of backlogged users drops $(i<n)$, which means that all contending users were correctly detected; (2) when the number of backlogged users increases $(i>n)$, which means that a detection error has occurred; and (3) when there is no change in the number of backlogged users $(i=n)$. In the latter case, two subcases can be further identified: one subcase when none of the idle users transmits while the backlogged users are not correctly detected, and the second subcase when none of the backlogged users transmits while all idle users that transmitted a packet are correctly detected. These cases can be written in mathematical form as

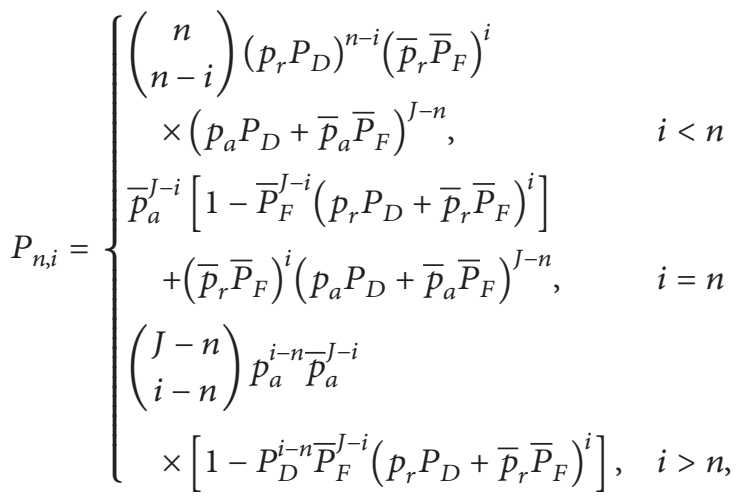

where $\overline{(\cdot)}=1-(\cdot)$. Since the probabilities in (8) can be easily derived by using the concepts of the binomial theorem or by referring to previous works on NDMA (e.g., $[5,6,12]$ ), their explicit derivation will be omitted here. The values of $P_{n, i}$ from (8) can be rearranged in a matrix array $\mathbf{P}$, which defines the following Markov model characteristic equation:

$$
\mathbf{P} \vec{\pi}=\vec{\pi},
$$

where $\vec{\pi}=[\pi(0), \ldots, \pi(J)]^{T}$ is the vector of steady-state probabilities of the network. Each element $\pi(n)$ indicates the probability of the network being in the $n$th state or having $n$ backlogged users. This equation can be solved by using standard eigenvalue techniques or iterative algorithms [14]. Once the vector of steady-state probabilities $\vec{\pi}$ has been obtained, it is possible to calculate other relevant performance metrics by simply averaging over the calculated probability space. The average number of users in the backlog state is thus given by

$$
E[n]=\sum_{n=0}^{J} n \pi(n)
$$

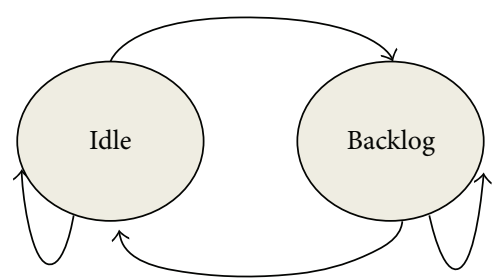

Figure 2: State model for each user in the network.

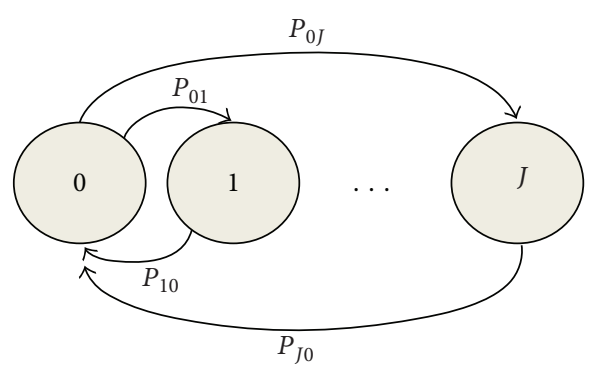

FIGURE 3: Network states and transition probabilities.

where $E[\cdot]$ indicates the statistical average operator. The average number of users in the backlog state is an indicator of the stability properties of the algorithm. The higher this indicator is, then the more the stability of the algorithm is degraded, as the users spend most of the time trying to retransmit a lost packet rather than attempting the transmission of new incoming packets.

4.2. Packet Throughput. The average number of packets correctly transmitted per epoch-slot can be obtained by averaging over the calculated probability space all the possible cases when all the users (both idle and backlogged) are correctly detected. This can be written as follows:

$$
S=\sum_{n=0}^{J} \pi(n) S(n)
$$

where

$$
\begin{aligned}
S(n)= & n\left(p_{r} P_{D}\right)\left(p_{r} P_{D}+\bar{p}_{r} \bar{P}_{F}\right)^{n-1}\left(p_{a} P_{D}+\bar{p}_{a} \bar{P}_{F}\right)^{J-n} \\
& +(J-n)\left(p_{a} P_{D}\right)\left(p_{a} P_{D}+\bar{p}_{a} \bar{P}_{F}\right)^{J-n-1} \\
& \times\left(p_{r} P_{D}+\bar{p}_{r} \bar{P}_{F}\right)^{n} .
\end{aligned}
$$

The first term in the previous expression contains the contribution of a backlogged user when the remaining users (either backlogged or nonbacklogged) are all correctly detected (either as active in case they transmitted or as inactive in case they did not transmit) with probability $\left(p_{r} P_{D}+\bar{p}_{r} \bar{P}_{F}\right)^{n-1}\left(p_{a} P_{D}+\bar{p}_{a} \bar{P}_{F}\right)^{J-n}$. The second term is given by the contribution of a non-backlogged user. Similarly, the average length of an epoch slot can be calculated by considering all the possible combinations of contributions of users (both backlogged and idle) that are detected as active 
by the BS (either correctly detected with probability $P_{D}$ or due to false alarm with probability $P_{F}$ ). This can be expressed as follows:

$$
\begin{aligned}
E[l]=\sum_{n=0}^{J} \pi(n) & {\left[\sum_{k=0 q=0}^{n} \sum^{J-n}\left(\begin{array}{l}
n \\
k
\end{array}\right)\left(\begin{array}{c}
J-n \\
q
\end{array}\right)\left\lceil\frac{k+q}{M}\right\rceil\right.} \\
& \left.\times P_{A, r}^{k} \bar{P}_{A, r}^{n-k} P_{A, a}^{q} \bar{P}_{A, a}^{J-n-q}+\bar{P}_{A, a}^{J-n} \bar{P}_{A, r}^{n}\right],
\end{aligned}
$$

where $k$ is the index for backlogged users, $q$ is the index for idle users, $P_{A, r}=p_{r} P_{D}+\bar{p}_{r} P_{F}$ is the probability of a backlogged user being detected as active, $P_{A, a}=p_{a} P_{D}+\bar{p}_{a} P_{F}$ is the probability of an idle user being detected as active, and the second term is due to the contribution of one time slot when none of the users is detected as active with probability $\bar{P}_{A, a}^{J-n} \bar{P}_{A, r}^{n}$. Having defined the terms $S$ and $E[l]$, it is now possible to define the throughput of the system as the longterm ratio of packets correctly received to the number of time slots used in the measurement. This can also be expressed as the ratio of the average number of packets correctly received to the average length of an epoch slot [12]:

$$
T=\frac{S}{E[l]} .
$$

4.3. Delay. The average backlog delay $\left(D_{b}\right)$, which can be defined as the average number of epochs that a user spends in the backlog state, can be calculated, using an extension of Little's theorem, as the ratio of the average number of backlogged users to the outgoing traffic [14]:

$$
D_{b}=\frac{E[n]}{S} .
$$

The total average delay of the system $(D)$ can then be expressed as the product of the backlog delay $D_{b}$ from (15) and a term that considers both service and queuing delay due to the use of more than one time slot in the transmission of packets in NDMA. The second term is commonly modeled in the literature of NDMA by the M/G/1 queue delay formula as used in $[5,11]$. Therefore, the total delay can be expressed as

$$
D=D_{b}\left(E\left[l_{r}\right]+\frac{E\left[l_{r}^{2}\right]}{2\left(1-\lambda E\left[l_{r}\right]\right)}+\frac{E\left[l_{i r}\right]}{2 E\left[l_{i r}^{2}\right]}\right),
$$

where $\lambda$ is the total incoming traffic per user while $E\left[l_{r}\right]$, $E\left[l_{r}^{2}\right], E\left[l_{i r}\right]$, and $E\left[l_{i r}^{2}\right]$ are the first- and second-order moments of the relevant and irrelevant epochs, respectively. The total incoming traffic $\lambda$ per user can be estimated using a traffic balance equation between the incoming and outgoing traffic per user [11]:

$$
p=\lambda E[l],
$$

where $p$ is the total transmission probability of a user, and which can be further expressed as the contribution of idle and backlog states:

$$
p=p_{i} p_{a}+p_{b} p_{r}
$$

where $p_{i}=\sum_{n=0}^{J} \pi(n)(1-n / J)$ is the average probability of a user being in the idle state and $p_{b}=\sum_{n=0}^{J} \pi(n)(n / J)$ is the average probability of a user being in the backlog state. To facilitate the derivation of expressions for the different types of epoch in (16), let us define the following auxiliary functions where we consider whether a given user under analysis contributes or not to the epoch length. The following function considers all the contributions to the length of an epoch given $x$ backlogged users have transmitted and only $z$ have been detected as active:

$$
\begin{aligned}
F_{a}(x, z)= & \sum_{n=x}^{J} \pi(n) p_{b}(n) \sum_{k=0}^{n-x} \sum_{q=0}^{J-n}\left(\begin{array}{l}
n \\
k
\end{array}\right)\left(\begin{array}{c}
J-n \\
q
\end{array}\right) \\
& \times\left\lceil\frac{k+q+z}{M}\right\rceil P_{A, r}^{k} \bar{P}_{A, r}^{n-k} P_{A, a}^{q} \bar{P}_{A, a}^{J-n-q},
\end{aligned}
$$

where $p_{b}(n)=n / J$ is the probability of a user being in the backlog state given $n$ users are in the backlog state. Similarly, the following function considers all the contributions to the length of an epoch given $y$ idle users have transmitted and only $z$ have been detected as active:

$$
\begin{aligned}
G_{a}(y, z)= & \sum_{n=0}^{J-y} \pi(n) p_{i} \sum_{k=0}^{n} \sum_{q=0}^{J-y-n}\left(\begin{array}{l}
n \\
k
\end{array}\right)\left(\begin{array}{c}
J-y-n \\
q
\end{array}\right) \\
& \times\left\lceil\frac{k+q+z}{M}\right\rceil P_{A, r}^{k} \bar{P}_{A, r}^{n-k} P_{A, a}^{q} \bar{P}_{A, a}^{J-y-n-q},
\end{aligned}
$$

where $p_{i}(n)=1-p_{b}(n)$ is the probability of a user being in the idle state given $n$ users are in the backlog state. Since the previous expressions only consider active contributions (i.e., users that are detected as active) to an epoch distribution, it is necessary to formulate two additional auxiliary functions to include those cases where no user is detected as active, thereby contributing with only one time slot to the epoch length. This can be expressed as

$$
F_{i}(x)=\sum_{n=x}^{J-x} \pi(n) p_{b}(n) \bar{P}_{A, a}^{J-n} \bar{P}_{A, r}^{n},
$$

for the case where $x$ backlogged users have transmitted, and

$$
G_{i}(y)=\sum_{n=0}^{J-y} \pi(n) p_{i}(n) \bar{P}_{A, a}^{J-n} \bar{P}_{A, r}^{n},
$$

for the case where $y$ idle users have transmitted. Let us now provide the desired expression for the first-order moment of the length of a relevant epoch in terms of the auxiliary functions defined in (19)-(22). First, we must consider that a given contending user can be either in the idle or in the backlog state. In addition, it can also be either correctly detected with probability $P_{D}$ (thereby contributing to the length of the epoch) or incorrectly detected as nonactive with probability $\bar{P}_{D}$ (thereby no contributing to the epoch length). 
The desired expression is thus given by the combination of all these possible detection cases as follows:

$$
\begin{aligned}
E\left[l_{r}\right]= & E[l \mid j \in \mathscr{T}]=P_{D}\left[F_{a}(1,1)+G_{a}(1,1)\right] \\
& +\bar{P}_{D}\left(\left[F_{a}(1,0)+F_{i}(1)+G_{a}(1,0)+G_{i}(1)\right]\right) .
\end{aligned}
$$

The expression for the irrelevant epoch can be simply obtained by replacing in the previous expression the term $P_{D}$ by $P_{F}$. Second-order moments are obtained in a similar way by using the squared length of the epoch in the auxiliary functions in (19)-(22). This concludes the derivation of the expressions for the total average delay.

\section{Results}

Let us now present some results that will illustrate the behavior of the analytical expressions derived in this paper using different parameters of the system. In all cases, the probability of a false alarm has been set to a fixed value of $P_{F}=0.001$ and the probability of detection is calculated according to the ROC model given in Section 3, particularly in (6) for $P_{F}$ and $P_{D}$, and using different values of signalto-noise ratio $\mathrm{SNR}=\sigma_{a}^{2} / \sigma_{v}^{2}$. In addition, for purposes of comparison, all the figures have included the results of the S-ALOHA protocol with the same number of antennas and same channel conditions. Figure 4 displays the steadystate probability distribution $\vec{\pi}$ from (9) for the different protocols using three different numbers of antennas $M=1$, $M=2$, and $M=4$ with an operational SNR of $7 \mathrm{~dB}$. In addition, the results were calculated using a packet transmission probability for idle users of $p_{a}=0.2$ and a packet retransmission probability of $p_{r}=0.01$ for the backlogged users. It can be observed that in all the cases the NDMA protocol presents a distribution of backlogged users shifted to the left side, which means that a low number of users remain, on average, in the backlog state. By contrast, the ALOHA protocol always stays at the right side of the figure. This means that NDMA reduces the number of unsatisfied users in the network, thereby presenting better stability features. Figure 5 displays the steady-state probability distribution of backlog states for the same configuration used in Figure 4, except for the packet transmission probability for idle users which is now set to $p_{a}=0.5$. Since the input traffic in this case is higher, the stability of all schemes has been considerably affected by showing a shift to the right side, that is, more backlogged users, in comparison with Figure 4 . The NDMA protocol can still be seen as the best solution in the left-most side of Figure 5. Figure 6 displays the steady-state probability distribution of backlog states for the same configuration used in Figure 4, but this time using SNR $=-3 \mathrm{~dB}$. The lower SNR conditions have affected the proposed algorithm and the reference ALOHA algorithm in different ways. The NDMA protocol with $M=4$ antennas is still the best with the lowest number of backlogged users. However, the second best scheme is now the S-ALOHA protocol with $M=4$ antennas, in contrast with the results in Figure 4 where NDMA with $M=2$ was the second best scheme. The NDMA protocol

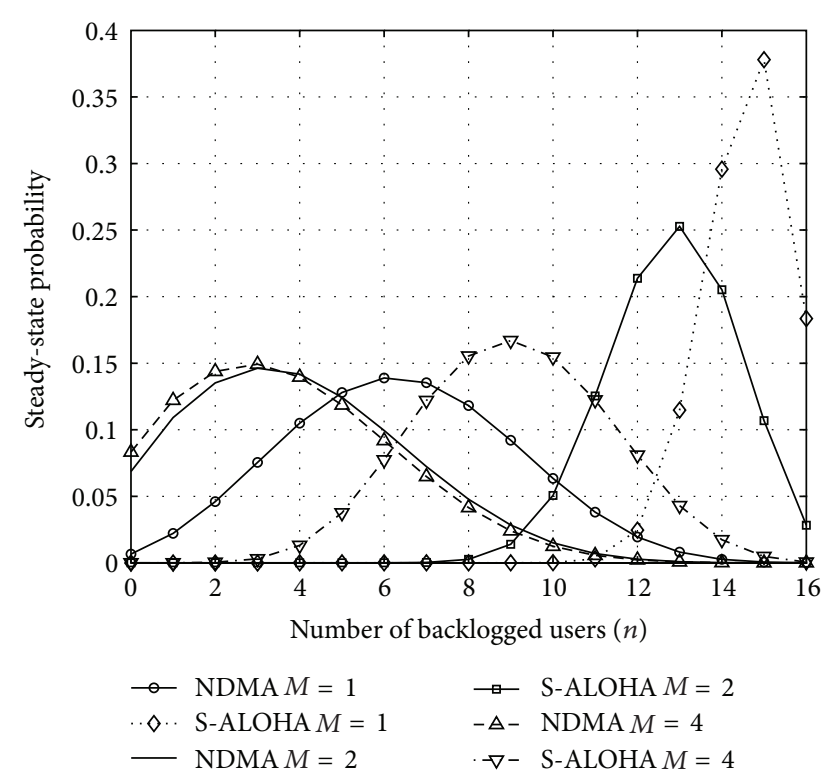

Figure 4: Steady-state probability distribution $\pi(n)$ of backlogged users with $p_{a}=0.2, p_{r}=0.01$, and $\mathrm{SNR}=7 \mathrm{~dB}$.

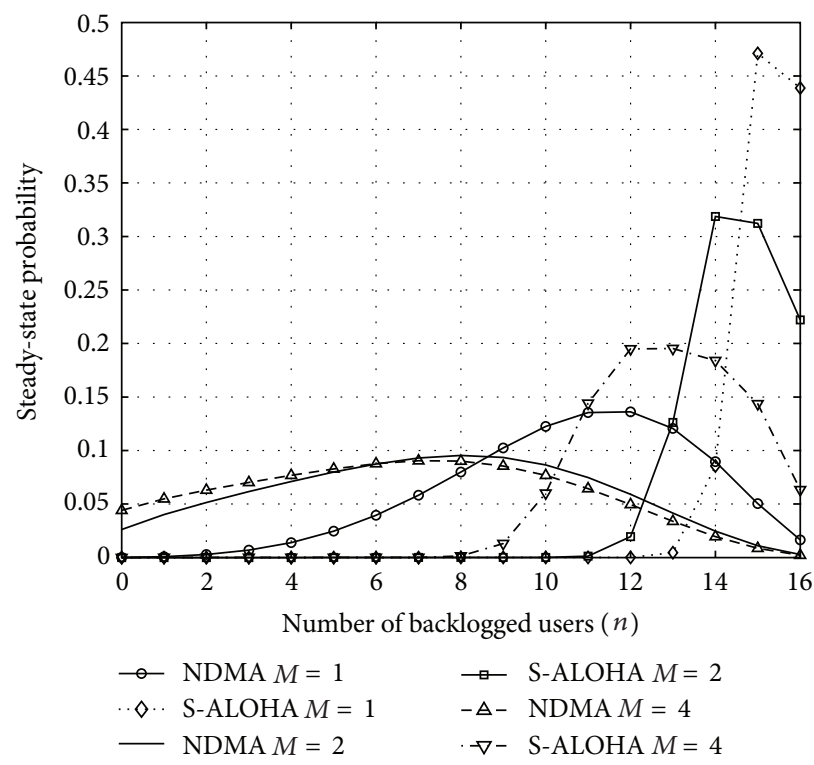

FIGURE 5: Steady-state probability distribution $\pi(n)$ of backlogged users with $p_{a}=0.5, p_{r}=0.01$, and $\mathrm{SNR}=7 \mathrm{~dB}$.

with $M<4$ now tends to behave as its ALOHA counterparts, which indicates that at low SNR values the performance of NDMA asymptotically degrades to ALOHA.

The results for throughput in Figure 7 using (14) and the total average delay performance in Figure 8 using (15) were calculated using a fixed value of packet transmission probability of idle users of $p_{a}=0.2$, an operational SNR of $\mathrm{SNR}=-3 \mathrm{~dB}$, and considering various values of the retransmission probability of backlogged users $p_{r}$. The case with $\mathrm{SNR}=7 \mathrm{~dB}$ is illustrated in Figures 9 and 10. In both results, it can be observed that NDMA always outperforms its 


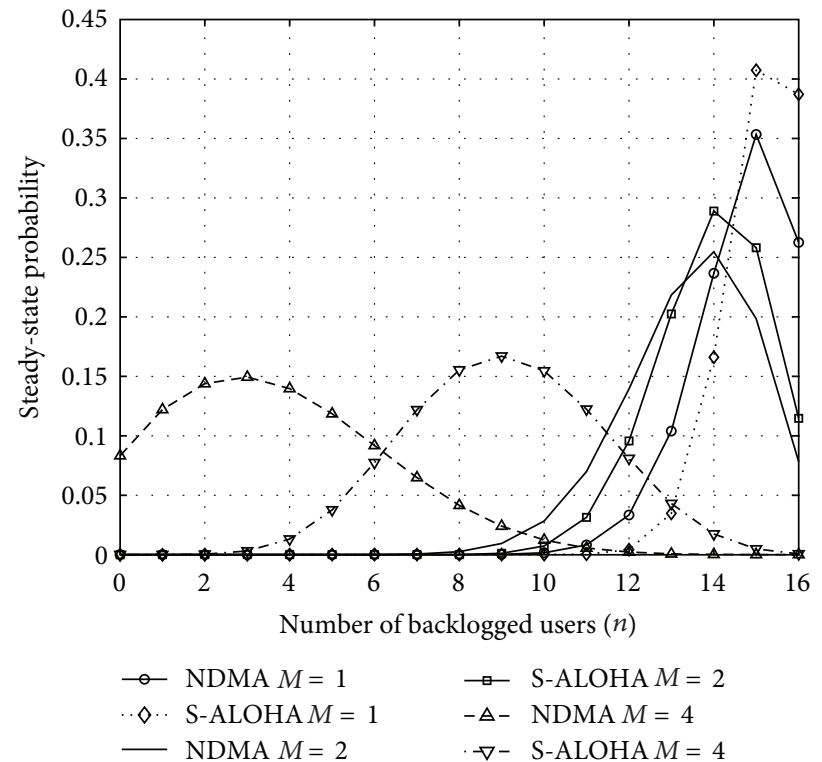

FIGURE 6: Steady state-probability distribution $\pi(n)$ of backlogged users with $p_{a}=0.2, p_{r}=0.01$, and SNR $=-3 \mathrm{~dB}$.

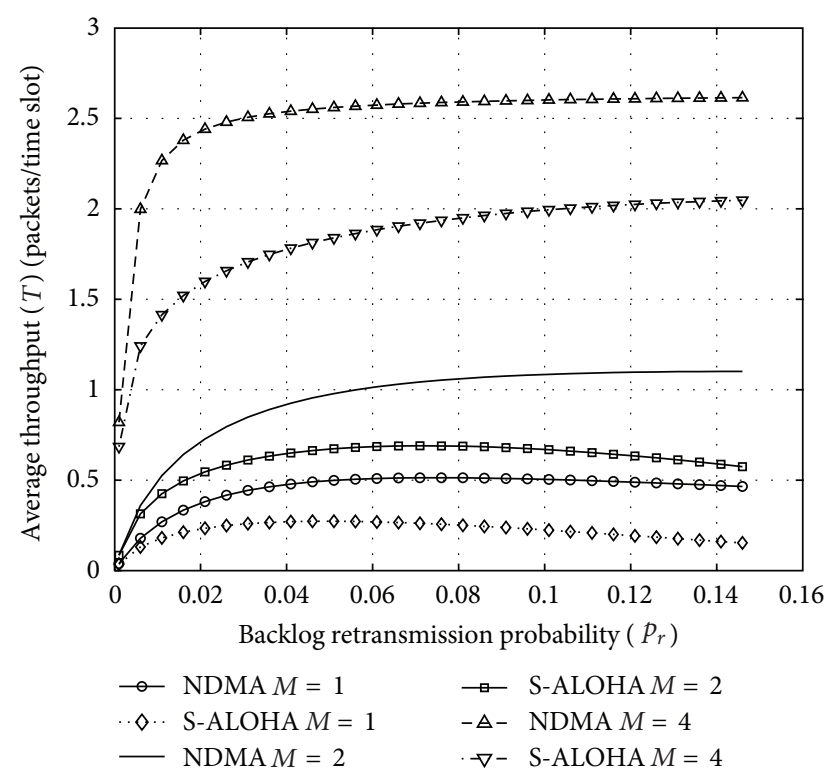

FIGURE 7: Average throughput $(T)$ versus backlog retransmission probability $\left(p_{r}\right)$ using $p_{a}=0.2$ and $\mathrm{SNR}=-3 \mathrm{~dB}$.

ALOHA counterparts. It can be observed, however, that for the particular case of $M=1$ and SNR $=-3 \mathrm{~dB}$ the NDMA protocol exhibits a slight decline in throughput for relatively high values of $p_{r}$. This indicates that at low SNR values, NDMA with $M=1$ needs a stabilization/optimization technique by selecting an appropriate value for $p_{r}$. In all other cases with $M=2,4$ antennas, and $\mathrm{SNR}=-3 \mathrm{~dB}$ the performance remains considerably smoother, which indicates that stabilization/optimization is not as essential as with $M=1$. In terms of delay, NDMA exhibits only a slight improvement

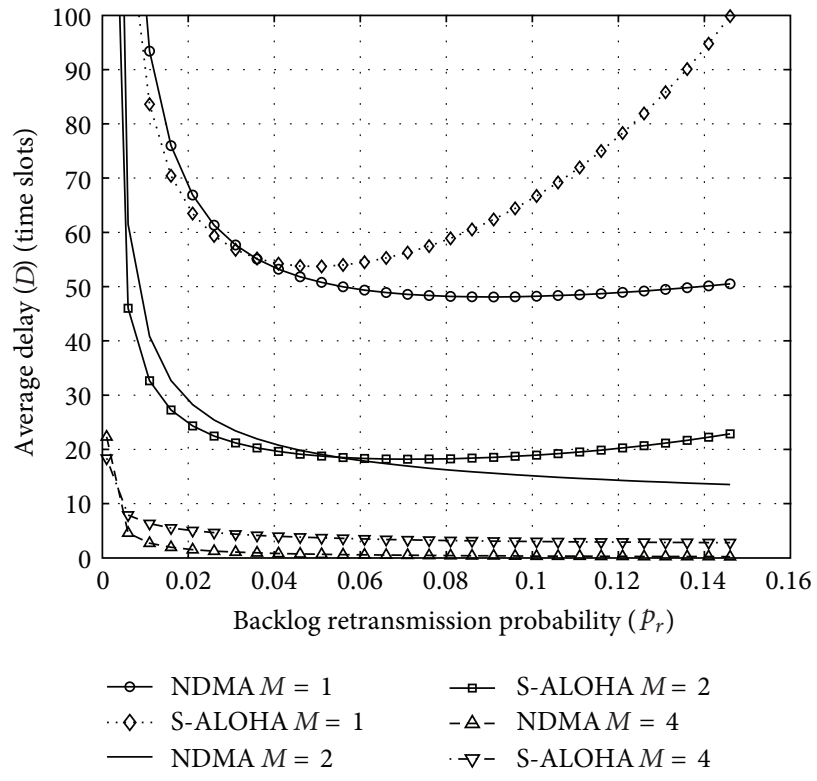

FIgURE 8: Average delay $(D)$ versus backlog retransmission probability $\left(p_{r}\right)$ using $p_{a}=0.2$ and $\mathrm{SNR}=-3 \mathrm{~dB}$.

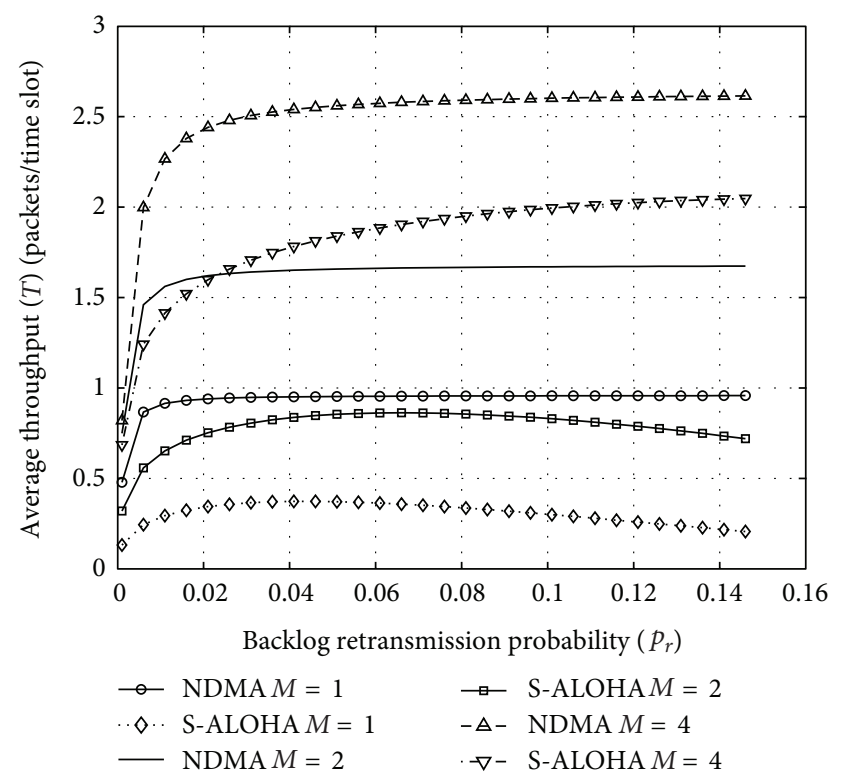

FIGURE 9: Average throughput $(T)$ versus backlog retransmission probability $\left(p_{r}\right)$ using $p_{a}=0.2$ and $\mathrm{SNR}=7 \mathrm{~dB}$.

over ALOHA in Figure 8 for low SNR. In the high SNR regime, however, delay reduction is considerable larger for all NDMA protocols with respect to their ALOHA counterparts (see Figure 10). In terms of throughput performance, NDMA at high SNR shows an almost flat feature for all values of $M$ (see Figure 9).

These results indicate that when SNR is low and when the number of antennas is low, NDMA and S-ALOHA exhibit similar stability characteristics, which also means that the stabilization techniques used for the ALOHA system should 


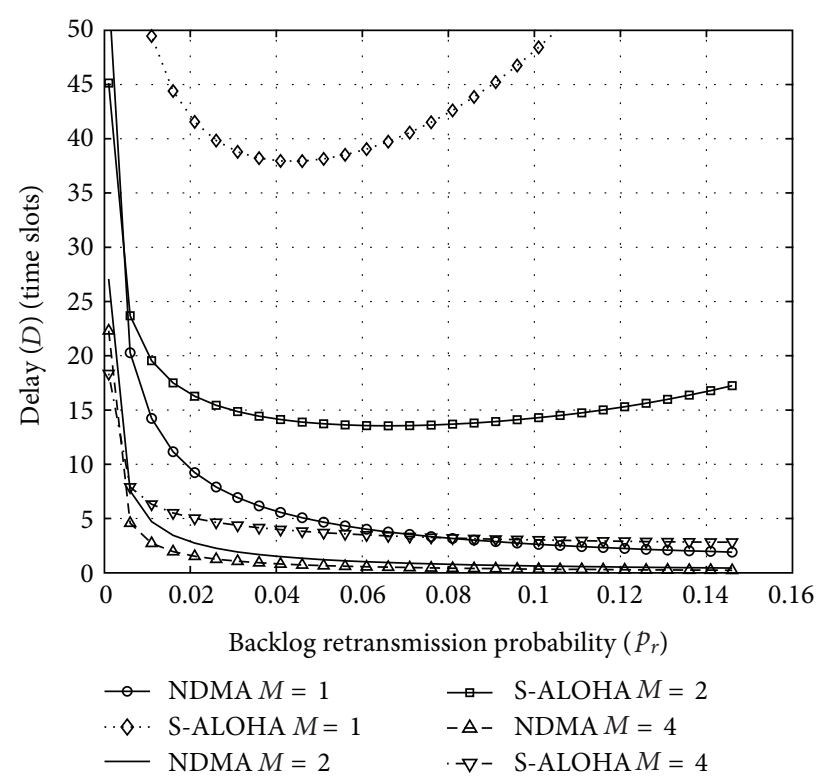

Figure 10: Average delay $(D)$ versus backlog retransmission probability $\left(p_{r}\right)$ using $p_{a}=0.2$ and $\mathrm{SNR}=7 \mathrm{~dB}$.

be applied to NDMA as well. By contrast, at high SNR or when a large number of antennas is used, the multiuser detection conditions considerably improve, thereby making NDMA very stable and without the need of a complex stabilization technique. This is an interesting result that shows how a strong PHY layer conveys the simplification of the MAC layer scheme for stabilization purposes. On the other hand, when the ability of the PHY layer declines, then more complex stabilization and in general MAC layer techniques will be required. Another interesting feature observed in the figures is that S-ALOHA and NDMA with $M=4$ seem unaltered when working at low and high SNRs (see Figures 7 and 9). This is due to the detector combining mechanism in (3) that exploits the diversity of the 4 antennas to produce more reliable detection statistics. This indicates that even with $\mathrm{SNR}=-3 \mathrm{~dB}$ the detector gain allows the system to mimic a higher SNR scenario.

\section{Conclusions}

This paper has presented the analysis of the dynamic properties (stability, backlog delay, and stable throughput) of a random access protocol jointly assisted by spatial and retransmission diversity. The paper used a Markov model that captures both queuing and channel statistics in the system. The results indicate that NDMA with multiple antennas considerably improves stability, throughput, and delay over all other considered solutions, particularly when multiuser detection conditions are relatively good. The modeling technique presented in this paper can be used and improved in the future to cope with more complex cross-layer optimization problems considering, for example, asymmetrical scenarios or systems with decentralized channel state information. The results in this paper also shed light on the interactions and dependencies between PHY and MAC layers. In particular, it was observed that when the PHY layer multiuser detection capabilities become strong, then the design of the MAC layer stabilization technique can be considerably simplified. On the contrary, when the PHY layer reduces its efficiency, it is necessary to design properly the MAC retransmission strategy for backlogged users so that the system behaves as desired. This conclusion can be useful for future investigations that address the joint performance of two or more layers of the OSI (open-system interconnection) model.

\section{References}

[1] L. Tong, V. Naware, and P. Venkitasubramaniam, "Signal processing in random access," IEEE Signal Processing Magazine, vol. 21, no. 5, pp. 29-39, 2004.

[2] M. H. Ngo and V. Krishnamurthy, "Game theoretic crosslayer transmission policies in multipacket reception wireless networks," IEEE Transactions on Signal Processing, vol. 55, no. 5 I, pp. 1911-1926, 2007.

[3] J. Crichigno, M. Y. Wu, S. Jayaweera, and W. Shu, “Throughput optimization in multihop wireless networks with multipacket reception and directional antennas," IEEE Transactions on Parallel and Distributed Systems, vol. 22, no. 7, pp. 1206-1213, 2011.

[4] G. Dimić, N. D. Sidiropoulos, and R. Zhang, "Medium access control-physical cross-layer design," IEEE Signal Processing Magazine, vol. 21, no. 5, pp. 40-50, 2004.

[5] M. K. Tsatsanis, R. Zhang, and S. Banerjee, "Network-assisted diversity for random access wireless networks," IEEE Transactions on Signal Processing, vol. 48, no. 3, pp. 702-711, 2000.

[6] R. Zhang and M. K. Tsatsanis, "Network-assisted diversity multiple access in dispersive channels," IEEE Transactions on Communications, vol. 50, no. 4, pp. 623-632, 2002.

[7] R. Zhang, N. D. Sidiropoulos, and M. K. Tsatsanis, "Collision resolution in packet radio networks using rotational invariance techniques," IEEE Transactions on Communications, vol. 50, no. 1, pp. 146-155, 2002.

[8] B. Ozgul and H. Deliç, "Wireless access with blind collisionmultiplicity detection and retransmission diversity for quasistatic channels," IEEE Transactions on Communications, vol. 54, no. 5, pp. 858-867, 2006.

[9] R. Samano-Robles, M. Ghogho, and D. C. McLernon, "A Multiaccess protocol assisted by retransmission diversity and multipacket reception," in Proceedings of the IEEE International Conference on Acoustics, Speech and Signal Processing (ICASSP '08), pp. 3005-3008, Las Vegas, Nev, USA, April 2008.

[10] R. Samano-Robles, M. Ghogho, and D. C. McLernon, "An infinite user model for random access protocols assisted by multipacket reception and retransmission diversity," in Proceedings of the IEEE 9th Workshop on Signal Processing Advances in Wireless Communications (SPAWC '08), pp. 111-115, July 2008.

[11] G. Dimić, N. D. Sidiropoulos, and L. Tassiulas, "Wireless networks with retransmission diversity access mechanisms: stable throughput and delay properties," IEEE Transactions on Signal Processing, vol. 51, no. 8, pp. 2019-2030, 2003.

[12] R. Samano-Robles, M. Ghogho, and D. C. McLernon, "Wireless networks with retransmission diversity and carrier-sense multiple access," IEEE Transactions on Signal Processing, vol. 57, no. 9, pp. 3722-3726, 2009. 
[13] Proakis, Digital Communications, McGraw Hill, 4th edition, 2001.

[14] F. A. Tobagi and L. Kleinrock, "Packet switching in radio channels: part IV-stability considerations and dynamic control in carrier sense multiple access," IEEE Transactions on Communications, vol. 25, no. 10, pp. 1103-1119, 1977. 

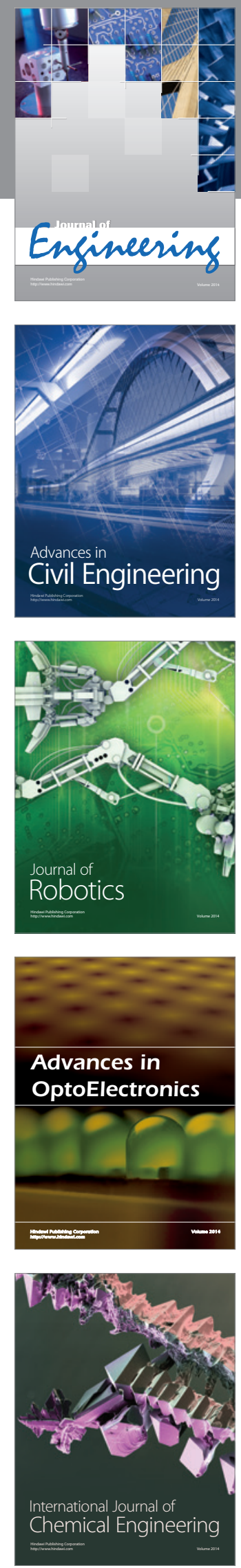

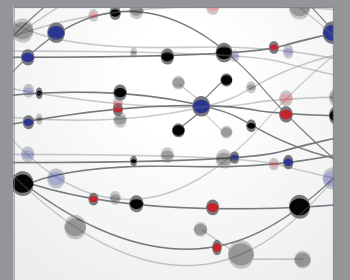

The Scientific World Journal
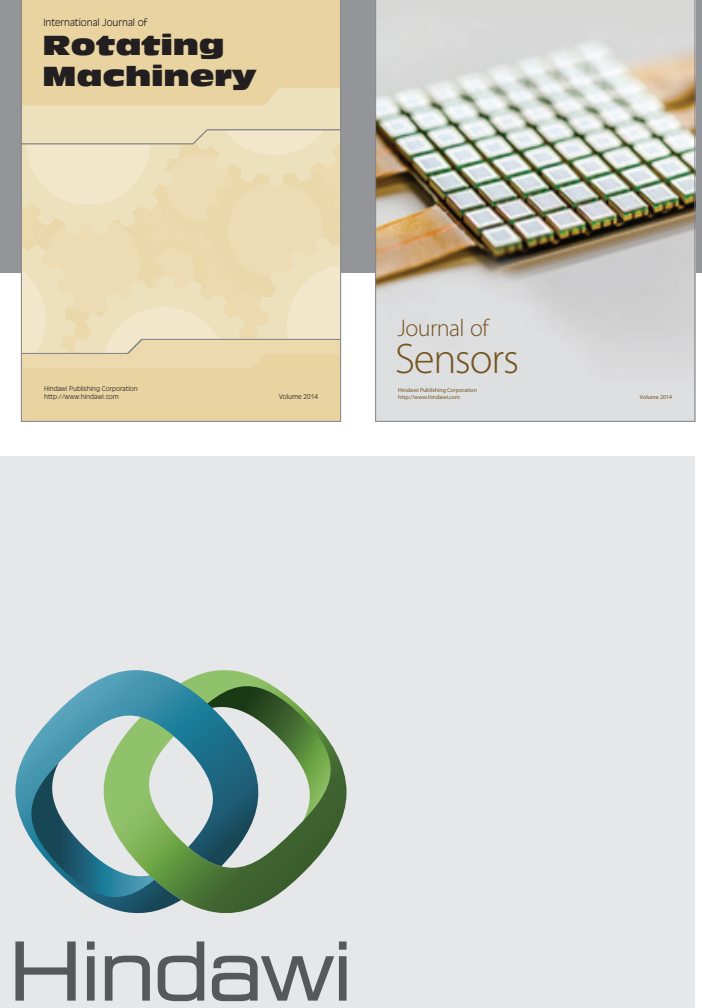

Submit your manuscripts at http://www.hindawi.com
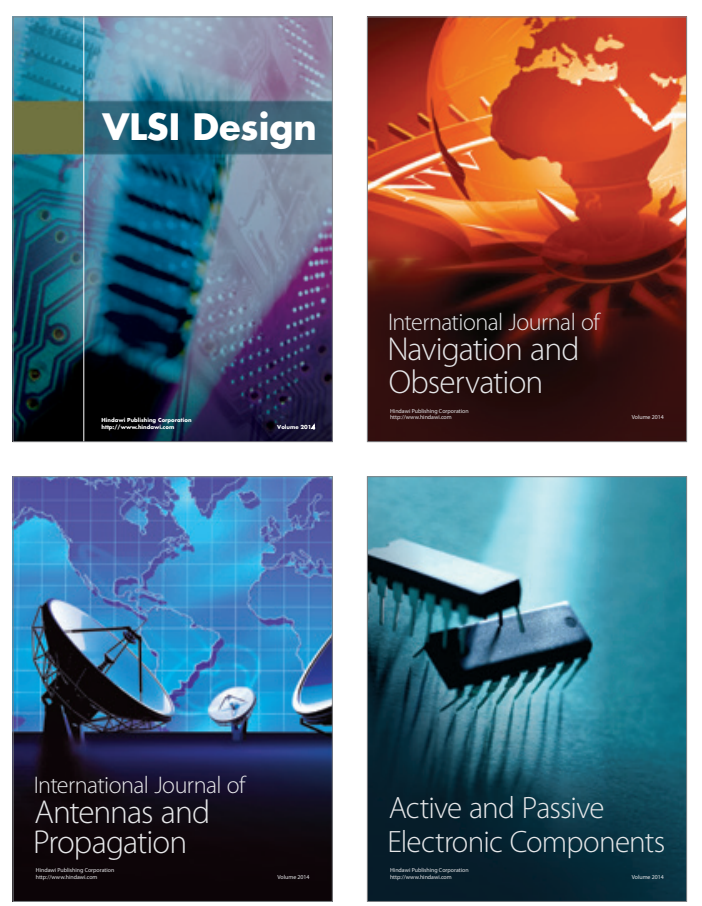
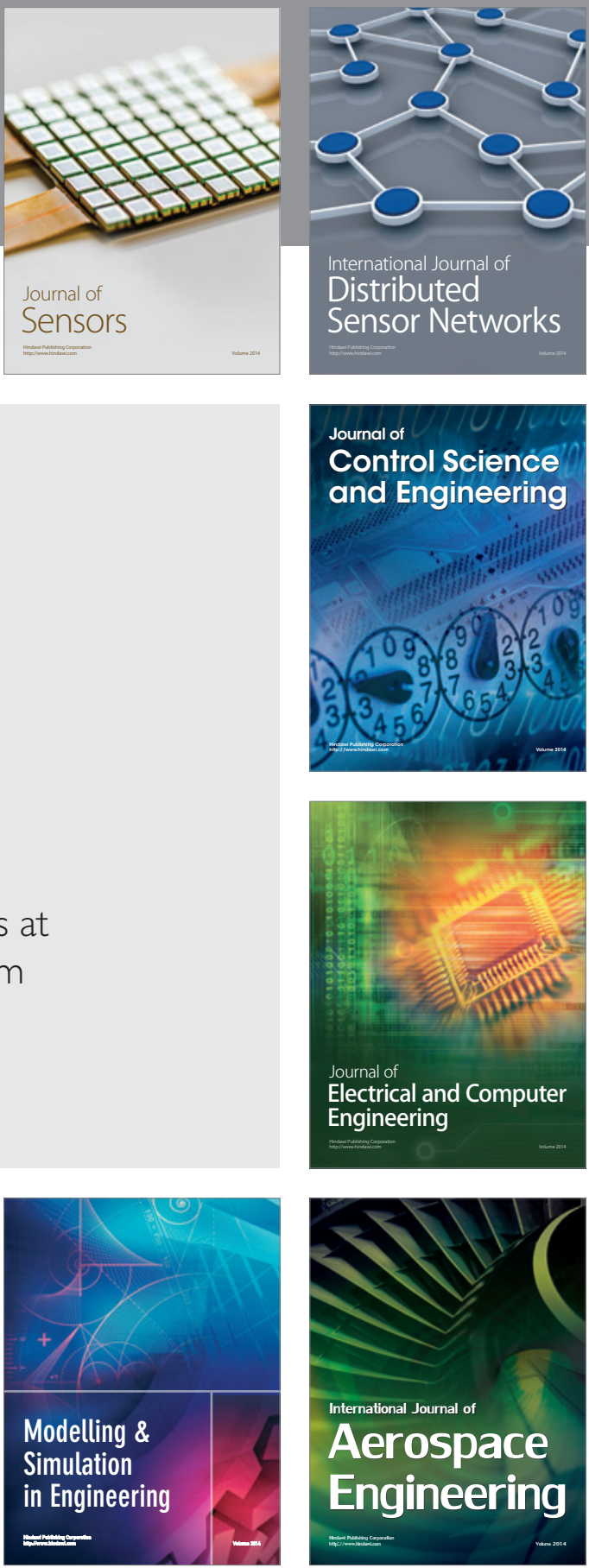

Journal of

Control Science

and Engineering
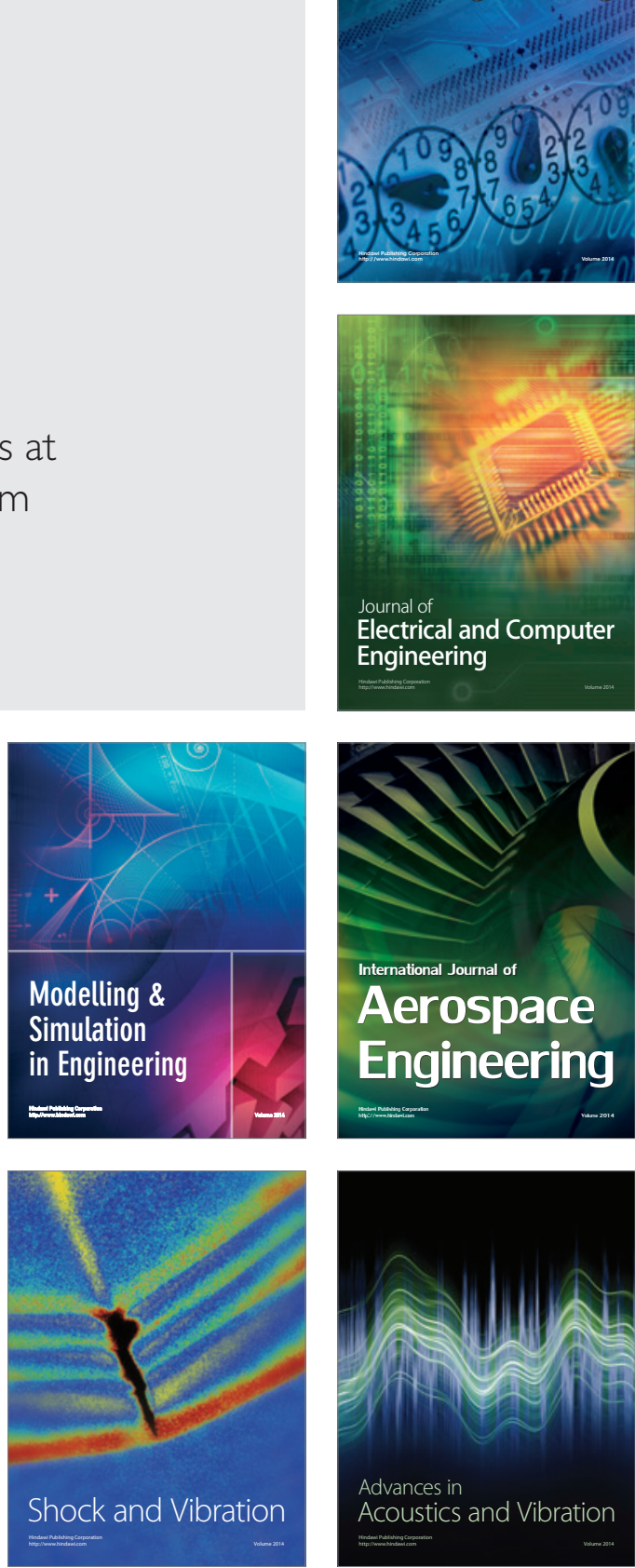